\title{
Dynamique de la redistribution d'un fond sédimentaire sous l'effet de nouvelles conditions de houle: une simulation de laboratoire.
}

\author{
Max Belzons \\ Maître de Conférences, IUSTI, UMR-139 du CNRS, Université de Provence \\ Vincent Rey \\ Maitre de Conférences, LSEET, URA 705 du CNRS, ISITV,Université de Toulon \\ et du Var
}

\section{Résumé}

La redistribution d'un fond sédimentaire sous l'effet de nouvelles conditions de houle est simulée dans un canal de laboratoire. Le fond de sédiment modéle, initialement horizontal, est tout d'abord érodé sous l'action d'une onde de surface monochromatique, partiellement stationnaire, jusqu'à atteindre un profil d'équilibre présentant des barres en modulation de Bragg. Puis on examine la redistribution de ce fond aprés avoir décalé l'onde de surface soit en phase, soit en fréquence. On observe dans tous les cas que le profil d'équilibre présente une modulation de Bragg, mais qu'il est atteint avec une dynamique fortement dépendante des conditions initiales.

\section{Introduction}

En zone littorale, la houle incidente interagissant fortement avec le fond et la plage, est d'une part atténuée et d'autre part partiellement réfléchie, ce qui constitue une protection naturelle de la côte. Dans le cas de littoraux sableux, l'onde partiellement stationnaire ainsi créée va alors évoluer avec le fond sédimentaire qu'elle érode, jusqu'à éventuellement atteindre un état d'équilibre dynamique qui semble correspondre au maximum de l'énergie réfléchie. L'état d'équilibre du fond prend parfois la forme remarquable d'un profil constitué de plusieurs barres de sable assez régulièrement espacées qui contribuent trés efficacement à réfléchir l'énergie de la houle incidente. Il est donc intéressant de comprendre l'évolution de ces barres sous l'effet de modifications des conditions de houle dues à l'altération d'un ou plusieurs paramètres de l'équilibre dynamique initial, tels que la direction, la hauteur, la fréquence de la houle incidente ou le coefficient de réflexion de la plage. L'étude que nous avons réalisée est la simulation en laboratoire de la formation de bartes par action d'une houle partiellement stationnaire, d'incidence normale, sur un fond sédimentaire, puis celle de l'évolution de ces barres par modification soit de la fréquence, soit de la phase de la houle incidente.

Aprés un rappel succint du dispositif expérimental et du modèle hydrodynamique, nous présentons les résultats expérimentaux de l'évolution de ces barres dans le cas d'un décalage de fréquence, puis dans le cas d'un décalage de phase, ainsi que la modélisation approchée du coefficient de réflexion du fond, mesuré en régime linéaire. 


\section{Dispositif expérimental et modèle théorique}

Les expériences ont été conduites dans un canal à houle de laboratoire qui en dépit de ses faibles dimensions (longueur $4.7 \mathrm{~m}$, largeur $39 \mathrm{~cm}$ et profondeur $15 \mathrm{~cm}$ ), s'est avéré apte à la modélisation de l'interaction d'ondes de gravité avec des fonds solides ou constitués de sédiments $[1,2]$. Ce canal dont les caractéristiques ont été présentées en détail dans [3] est maintenant équipé d'un batteur mû par un moteur linéaire ce qui permet la production de houles diverses et en particulier linéaires ou non, dont la fréquence du fondamental peut varier de 0.6 à $5 \mathrm{~Hz}$. Une plage d'inclinaison variable, située à l'autre extrémité du canal, produit le taux d'onde stationnaire requis. Dans la partie centrale du canal, le fond est formé d'un sédiment modèle constitué de billes de verre de diamètre $80 \mu \mathrm{m}+25 \mu \mathrm{m}$, préalablement décompacté et nivelé selon un plan horizontal à $\pm 0.5 \mathrm{~mm}$ prés. En amont et en aval, ce fond se prolonge par deux plaques de PVC horizontales et au même niveau, ce qui délimite la zone de mesure.

Deux écho-sondeurs à ultasons sont fixés sur un chariot mobile le long du canal. L'un mesure (généralement dans l'axe du canal) la topographie du fond, l'autre l'amplitude de l'onde de surface le long de ce fond avec, dans les deux cas, une résolution de $100 \mu \mathrm{m}$. la mesure de la topographie du fond se fait lorsque le batteur est arrêté et au bout d'un temps cumulé d'érosion $\Delta t$. Ces mesures très rapidement réalisées (moins d'une minute), peuvent être faites en nombre suffisant pour obtenir l'évolution de la topographie du fond depuis l'apparition des rides initiales jusqu'au profil ultime. Le temps de mesure de l'enveloppe de l'onde de surface étant plus long (de l'ordre de $5 \mathrm{mn}$ ), elle doit se faire avec une amplitude incidente suffisamment faible pour éviter que l'érosion ne se poursuive lors de la mesure. L'analyse harmonique de ce régime linéaire ou faiblement non linéaire, foumit, en particulier, le coefficient de réflexion du fond.

La modelisation de l'écoulement du fluide (vitesse, élongation de la surface libre,coefficient de réflexion,..) suppose un régime potentiel linéaire et un fond solide imperméable. Les expériences antérieures réalisées dans notre canal à houle ont montré que cette modélisation, proposée et décrite en détail dans [4], rend compte correctement du comportement général de l'écoulement malgré la présence d'effets dissipatifs et de faibles non linéarités et vorticité, aussi bien dans le cas de fonds solides $[1,5]$, que dans celui de fonds sédimentaires $[3,6]$. Dans le cas où le profil du fond est de faible amplitude et de faible pente, le modèle potentiel exact peut, selon la méthode variationnelle de Miles [7], être simplifié [8]. C'est cette modélisation que nous avons utilisée ici pour le coefficient de réflexion.

\section{Résultars expérimentaux.}

Les expériences d'érosion ont été conduites en eau de profondeur $6 \mathrm{~cm}$ et pour une onde incidente monochromatique dont la fréquence était soit $f_{0}=1.5 \mathrm{~Hz}$, soit $f_{1}=$ $1.8 \mathrm{~Hz}$, et l'amplitude a suffisante $(>15 \mathrm{~mm})$ pour que la vitesse tangentielle au voisinage immédiat de la couche limite visqueuse soit assez nettement au-dessus du seuil d'érosion. L'onde incidente avait ainsi une longueur d'onde $\lambda_{0}=46.5 \mathrm{~cm}$ pour $f_{0}$ et $\lambda_{1}=37.0 \mathrm{~cm}$ pour $f_{1}$. Le coefficient de réflexion de la plage était de 0.20 ou plus afin d'avoir une formation initiale de barres suffisamment rapide. Les mesure du coefficient de réflexion des profils ultimes ont été effectuées autour de la fréquence d'érosion, $f_{0}$ ou $f_{1}$, et avec une amplitude $a=5 \mathrm{~mm}$. La confrontation 
de ces résultats au modèle théorique exigeant la connaissance de module et de la phase du coefficient de réflexion de la plage, ces deux paramètres ont été préalablement déterminés à partir de la mesure de l'enveloppe de la vague sur le fond non érodé

\subsection{Effet d'un décalage de fréquence}

Dans un premier temps, le fond initialement plan est soumis à l'action de l'onde partiellement stationnaire de fréquence $\mathrm{f}_{0}=1.5 \mathrm{~Hz}$. On poursuit l'érosion pendant un temps $\Delta t$ jusqu'à ce que le fond soit en équilibre dynamique avec l'onde. Pour la série d'expériences retenue, l'enregistrement du profil médian de ce fond présente une modulation à grande échelle montrant 9 barres, la première étant sur le fond de PVC, d'espacement moyen proche de $\lambda_{\sigma} / 2$ (Fig. 1a). La résonance de Bragg entre l'onde et le fond que suggère cette modulation, est confirmée par la mesure du coefficient de réflexion $R(f)$ du fond en régime linéaire qui présente effectivement un maximum proche de la fréquence $f_{0}$. Ainsi pour le profil de la Fig. 1a, $R(f)$ est maximum à $1.45 \mathrm{~Hz}$ (Fig. 2). Dans un deuxième temps, le fond ainsi formé est soumis à l'action d'une onde d'amplitude comparable à la précédente, mais de fréquence $f_{1}=1.8 \mathrm{~Hz}$, jusqu'à atteindre le profil d'équilibre. Ainsi en érodant pendant $\Delta t=6 \mathrm{~h}$ le profil de la figure $1 \mathrm{a}$, on obtient le profil de la figure $1 \mathrm{~b}$ présentant 9 barres d'espacement moyen proche de $\lambda_{1} / 2$ et dont le coefficient de réflexion en régime linéaire est maximum pour $1.76 \mathrm{~Hz}$ (Fig. 3). Le fond modulé en $\lambda_{0} / 2$ sous

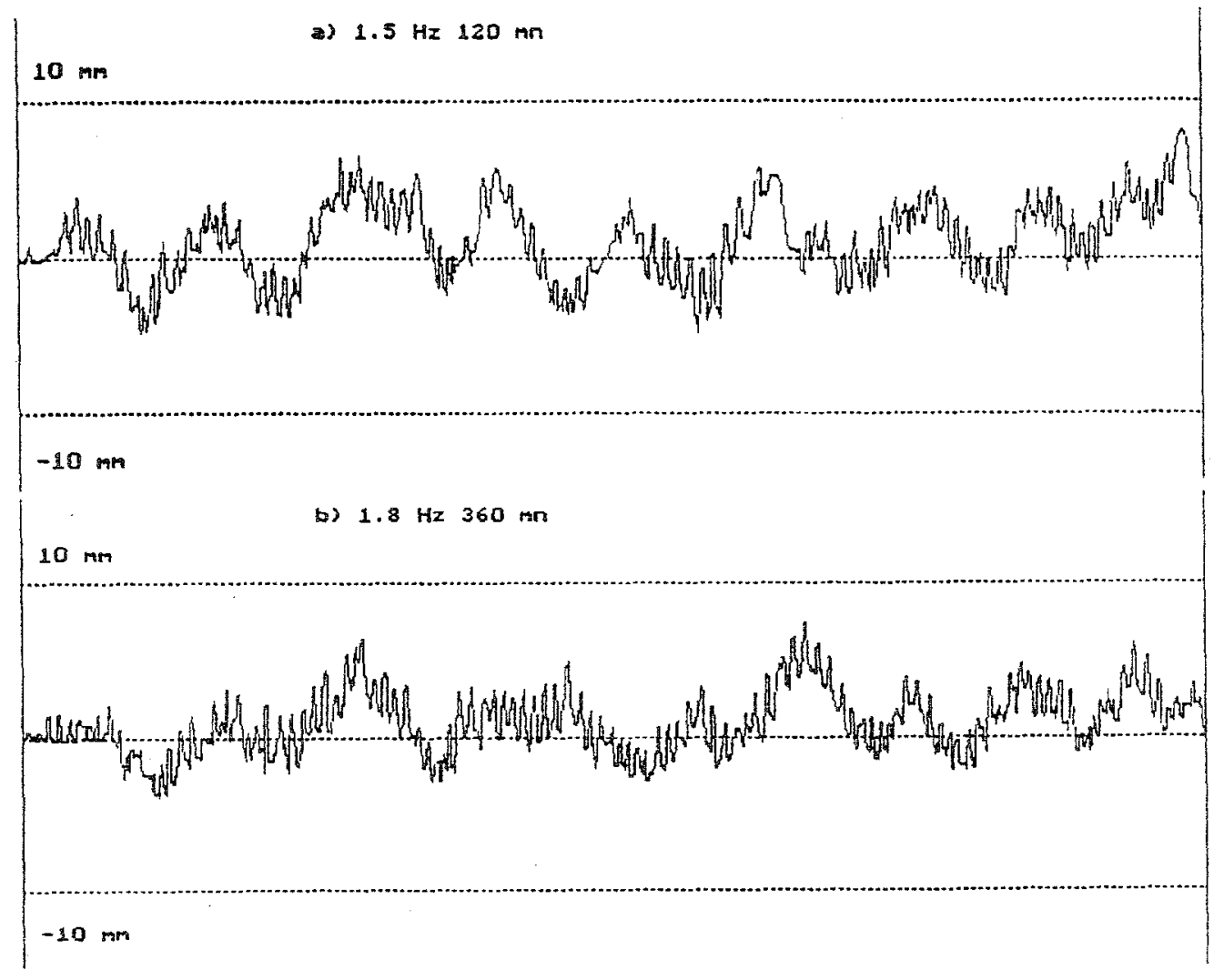

Fig. 1- Profils d'équilibre entre 0 et $196 \mathrm{~cm}$, avant et aprés décalage en fréquence. 
l'action de londe de fréquence $f_{0}$, est donc redistribué par l'action ultérieure de l'onde de fréquence $f_{1}$ selon une modulation en $\lambda_{1} / 2$ et donc une résonance de Bragg pour la fréquence $f_{1}$, mais avec une dynamique sensiblement plus lente que pour l'érosion initiale.

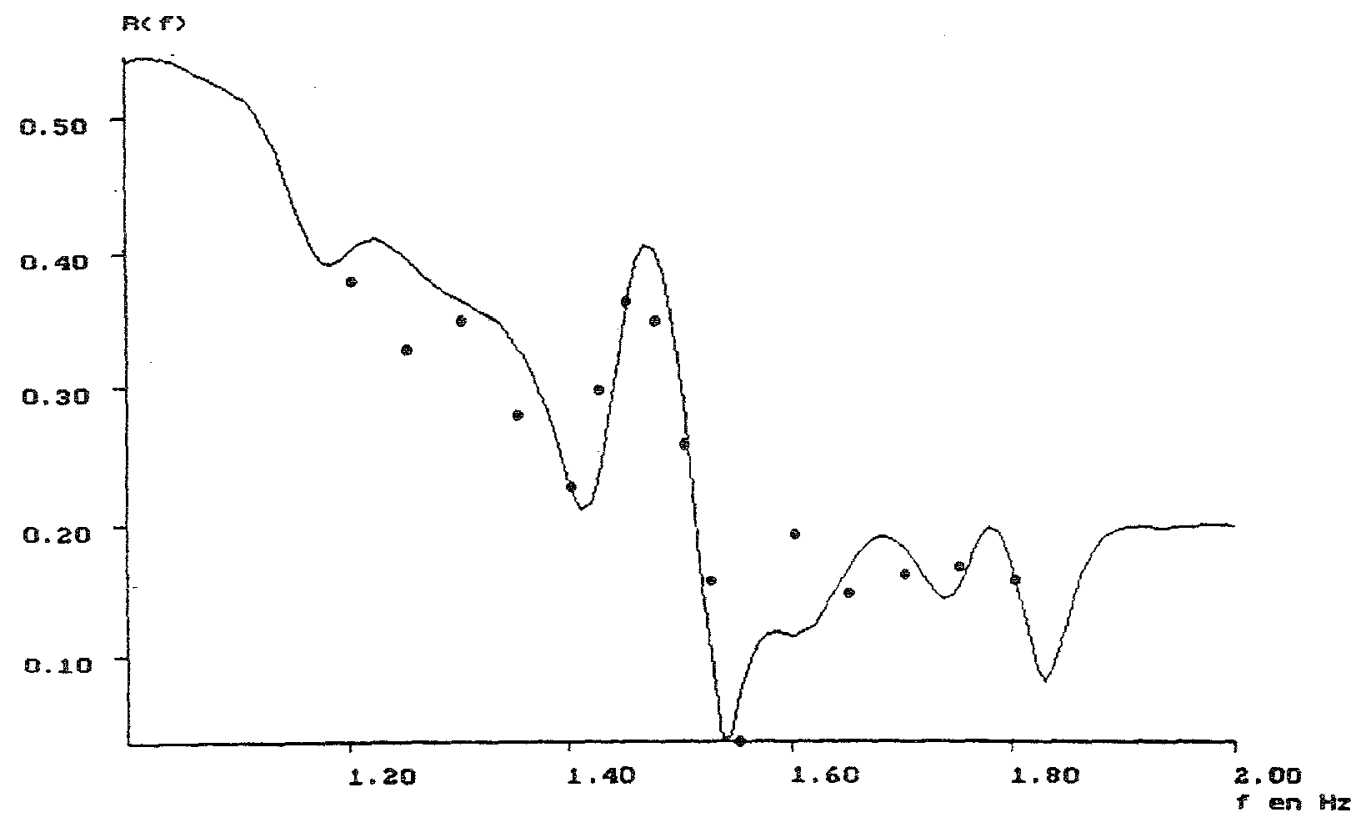

Fig. 2-Coefficient de réflexion du profil de la Fig. 1a): expérience ( $\bullet$ et calcul.

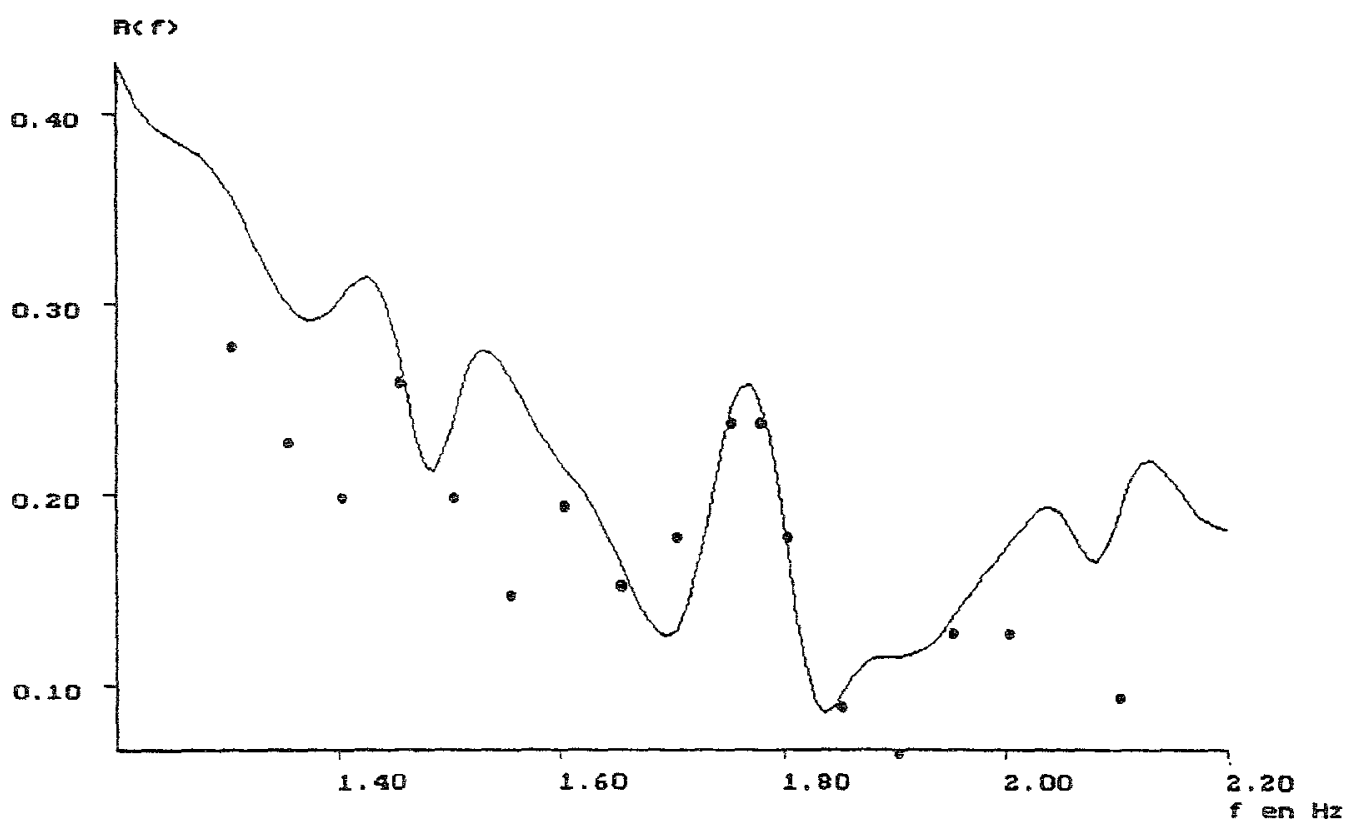

Fig. 3- Coefficient de réflexion du profil de la Fig. 1b): expérience ( $)$ et calcul. 


\subsection{Effet d'un décalage de phase}

Le fond initialement plan est tout d'abord érodé par l'onde de fréquence $f_{0}$ jusqu'à atteindre un profil d'équilibre présentant des barres d'espacement moyen proche de $\lambda_{0} / 2$. On poursuit ensuite l'érosion avec la même onde mais après avoir translaté la plage de $\lambda_{0} / 4$, soit en amont soit en aval, ce qui a pour effet de déphaser le champs de vagues de $\pm \pi / 2$ par rapport au fond. Ce déphasage peut être confirmé par enregistrement du champs de vagues avant et après translation de la plage.

Lors d'expériences préliminaires, on a pu constater qu'après translation de la plage de $\lambda_{0} / 4$ en amont, le profil d'équilibre initial est progressivement détruit, mais que l'on n'observe toujours pas de modulation en $\lambda_{\sigma} / 2$ même à $\Delta t=4$ ou $5 \mathrm{~h}$. Une autre série d'expériences semble cependant indiquer que cette modulation pourrait se faire avec une trés lente dynamique. Pour ces expériences, l'inclinaison de la plage a été augmentée et son coefficient de réflexion porté à 0.30 pour $\mathrm{f}_{0}$ de façon à obtenir des barres de plus grande amplitude lors de l'érosion initiale. La plage étant préalablement transiatée en amont de $\lambda_{0} / 4$ par rapport à sa position standard, le profil d'équilibre du fond, atteint à $\Delta t=90 \mathrm{mn}$, présente 8 fortes barres règulièrement espacées de $\lambda_{0} / 2$ (Fig. 4a). Après translation de la plage de $\lambda_{0} / 4$ en aval, l'érosion de ce fond initial est alors poursuivie pendant un temps trés long. Pour $\Delta t=25 \mathrm{~h}$, on observe une assez nette substitution crêtes-creux pour les barres mais avec une modulation du fond qui reste irrégulière (Fig. 4b).

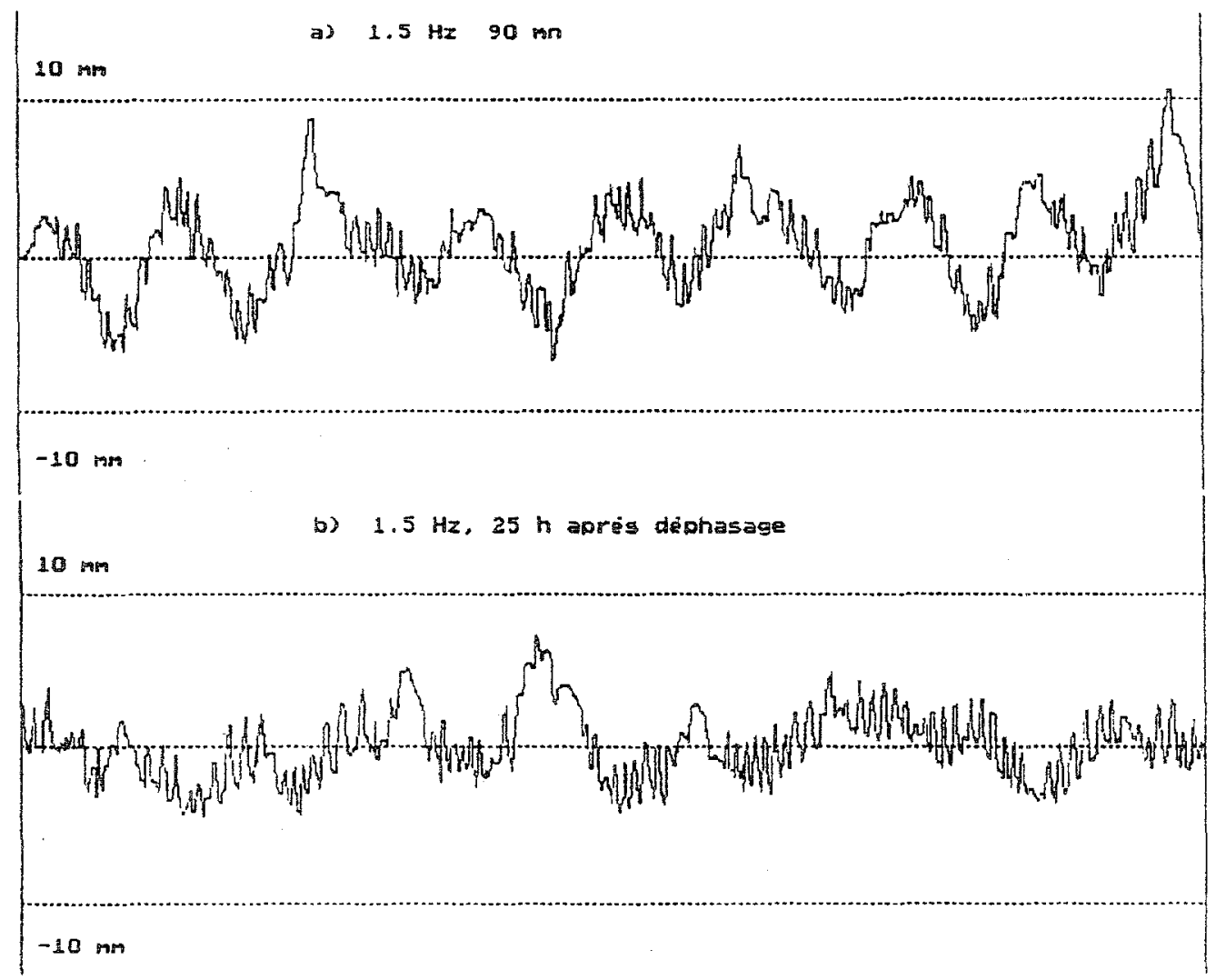

Fig. 4- Profils d'équilibre entre 0 et $196 \mathrm{~cm}$, avant et aprés déphasage de $\pi / 2$. 


\section{Discussions et conclusion}

Les expériences présentées ici semblent confirmer que sous l'action dune onde partiellement stationnaire de fréquence $f_{0}$, de longueur d'onde $\lambda_{0}$, un fond sédimentaire initialement horizontal, acquiert un profil d'équilibre par autoadaptation dynamique avec l'onde qui l'érode. Ce profil d'équilibre initial est généralement constitué de barres assez régulièrement espacées de $\lambda_{0} / 2$ et le facteur de réflexion en régime linéaire de ce fond présente un maximum marqué proche de la fréquence d'érosion $f_{0}$ (résonance de Bragg [9]).

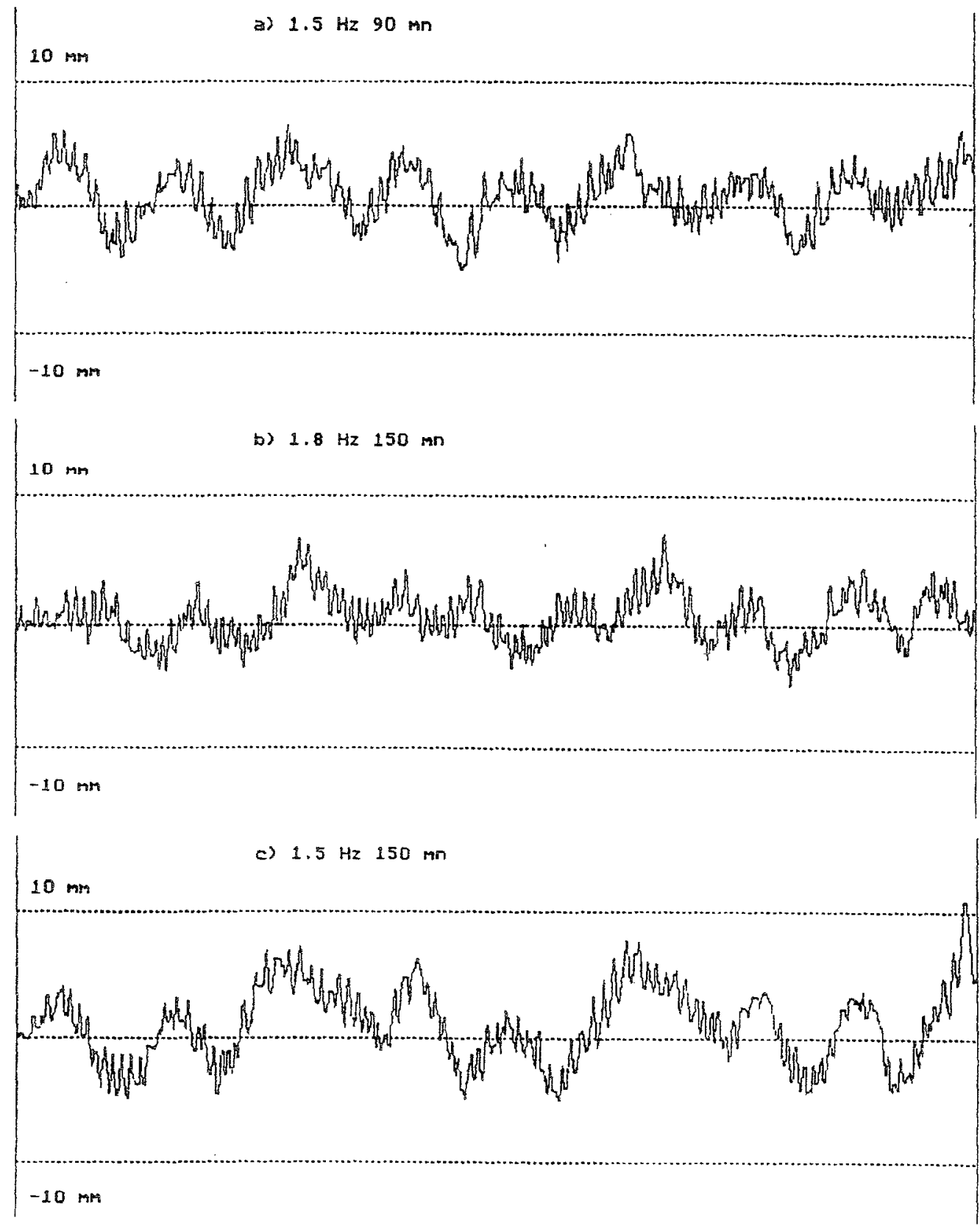

Fig. 5- Profils d'équilibre entre 0 et $196 \mathrm{~cm}$, avant et aprés décalage en fréquence puis aprés retour à la fréquence initiale. 
Cette étude montre surtout que sous l'action ultérieure d'une onde de fréquence $f_{1} \neq$ $f_{0}$ (décalage de fréquence), on observe une redistribution du fond initial, avec une modulation aux temps longs en $\lambda_{1} / 2$ et une résonance de Bragg vers $f_{1}$.

On observe donc une réadaptation du fond aux nouvelles conditions d'érosiontransport de sédiment, avec cependant une dynamique beaucoup plus lente que pour l'érosion initiale. Cette réadaptation est encore plus lente dans le cas d'une onde décalée en phase, et on n'observe pas clairement la translation de $\lambda_{0} / 4$ qui devrait être induite par celle du champs de vagues, mais cependant une assez nette substitution crêtes-creux des barres qui va dans le même sens. Pour ce qui concerne le redistribution d'un fond initial modulé en barres, l'efficacité d'un décalage de fréquence de l'onde est donc bien supérieure à celle d'un décalage de phase. Ces résultats montrent que l'ensemencement du fluide en particules de sable, processus à seuil fortement non linéaire, a une dynamique très dépendante des conditions initiales, contrairement aux effets à long terme du transport sédimentaire, dominés par la nature de l'écoulement hors couche limite et correctement décrits à partir d'une modélisation potentielle linéaire des ondes. Ainsi la relative indépendance du profil d'équilibre vis à vis des conditions initiales peut être confirmée par une expérience de double décalage de fréquence. Dans un premier temps, le fond initialement horizontal, érodé par l'onde de fréquence $\mathrm{f}_{0}$, atteint son profil d'équilibre en $\Delta t=90 \mathrm{mn}$ (Fig. 5a). Puis ce profil d'équilibre initial modulé en $\lambda_{0} / 2$, subit l'action ultérieure de l'onde de fréquence $f_{1}$ et atteint son profil d'équilibre en $\Delta t=150 \mathrm{mn}$ (Fig. $5 b$ ). Enfin, ce nouveau profil initial maintenant modulé en $\lambda_{1} / 2$, est ultérieurement érodé par l'onde de fréquence $f_{0}$ et on constate (Fig. $5 \mathrm{c}$ ) que le profil d'équilibre atteint à $\Delta t=150 \mathrm{mn}$ est trés proche du profil d'équilibre initial.

De même l'aptitude du modèle potentiel approché à décrire la résonance de Bragg est démontrée en comparant les calculs aux résultats expérimentaux du coefficient de réflexion en régime linéaire $R(f)$. Pour les 2 profils d'équilibre pour lesquels $R(f)$ a été mesuré, on a calculé le coefficient de réflexion pour le fond ayant comme génératrice le profil correspondant et avec les valeurs expérimentales du module et de la phase de l'onde réfléchie par la plage. On constate alors que le calcul (courbe en trait continu) rend compte assez correctement de la résonance de Bragg et du caractère fortement oscillant de $R(f)$ mis expérimentalement en évidence sur les figures 2 et 3 et vraisemblablement dû au faible nombre de barres ainsi qu'à leurs irrégularités.

Des expériences en cours devraient permettre de préciser la dynamique de cette redistribution sédimentaire, et en particulier sous l'effet de modifications de condition de houle plus diverses.

\section{Références bibliographiques}

[1] Rey V., Belzons M., and Guazzelli E.,1992. Propagation of surface gravity waves over a rectangular submerged bar, J.Fluid Mech. 235, 453-479.

[2] Rey V., Davies A.G. and Belzons M., 1995. On the formation of bars by the action of waves on an erodible bed: a laboratory study, J. Coastal Research, 11 , 4, 1180-1194. 
[3] Rey V., 1991. Propagation d'ondes de gravité au dessus de fonds solides ou constitués de sédiments: application à l'étude d'interactions dynamiques ondessédiments, Thèse de lUniversité de Provence, Marseille.

[4] Rey V., 1992. Propagation and local behaviour of normally incident waves over varying topography, European Journal of Mechanics B/Fluids, 11, 213-232.

[5] Belzons M., Rey V. and Guazzelli E., 1991. Subharmonic Bragg resonance for surface water waves, Europhysics Letters, 16(2), 189-194.

[6] Belzons M. et Rey V., 1995. Réflexion de la houle par un obstacle immergé sur un fond érodable. $5^{\circ}$ Journées de l'hydrodynamique, Rouen, 101-114.

[7] Miles W.J, 1967. Surface-wave scattering matrix for a shelf, J. Fluid Mech., 28(4), 755-767.

[8] OHare T.J. and Davies A.G., 1992. A new model for surface wave propagation over undulating topography. Coastal Engineering, 18, 251-266.

[9] Davies A.G and Heathershaw A.D, 1984. Surface wave propagation over sinusoidally varying topography. J. Fluid Mech., 144, 419-443. 